\title{
Associations of dipping and non-dipping hypertension with cardiovascular diseases in patients with dyslipidemia
}

Siping Dai, Bo Huang, Yunliang Zou, Yan Liu

Department of Emergency, The Third People's Hospital, Huizhou, China

Submitted: 8 October 2017

Accepted: 11 December 2017

Arch Med Sci 2019; 15, 2: 337-342

DOI: https://doi.org/10.5114/aoms.2018.72609

Copyright $\odot 2018$ Termedia \& Banach

\section{Abstract}

Introduction: Dyslipidemia combined with hypertension increases the risk of cardiovascular disease (CVD). The current study aimed to investigate the association of dipping and non-dipping hypertension with CVD in patients with dyslipidemia.

Material and methods: A total of 243 documented dyslipidemia patients with hypertension were enrolled. Clinical characteristics and clinic and 24hour blood pressure (BP) parameters were compared between dipping and non-dipping groups based on 24-hour ambulatory blood pressure monitoring. Logistic regression analysis was performed to evaluate the association of dipping and non-dipping hypertension with CVD.

Results: Compared to the dipping group, patients in the non-dipping group were older, more likely to be male and smokers, had higher serum creatinine levels, and were more likely to have chronic kidney disease and CVD ( $p<$ 0.05 for all comparisons). No significant between-group differences in clinic systolic and diastolic BP (SBP and DBP) were observed. However, compared to the dipping group, 24-hour SBP, nighttime SBP and DBP, and night-day ratio of SBP and DBP were all significantly higher in the non-dipping group ( $p<0.05$ for all comparisons). In the dipping group, only night-day ratio of SBP was significantly associated with CVD, with an odds ratio (OR) of 1.09 (95\% confidence interval $(\mathrm{Cl})$ of 1.02-1.34). In the non-dipping group, both night-day ratio of SBP and DBP were significantly associated with CVD, with an OR of 1.72 (95\% Cl: 1.33-2.06) and 1.23 (95\% Cl: 1.05-1.66), respectively. Conclusions: In patients with dyslipidemia, non-dipping hypertension is more closely related to CVD compared to dipping hypertension.

Key words: dyslipidemia, hypertension, cardiovascular diseases.

\section{Introduction}

Cardiovascular diseases (CVD) are a leading cause of morbidity and mortality around the world [1, 2]. Among the risk factors of CVD, dyslipidemia and hypertension are the most important ones [3-5]. It is reported that dyslipidemia combined with hypertension accounted for more than half of population attributable risks of CVD $[2,6]$. Therefore, it is clinically important to better manage hypertension in patients with dyslipidemia, so as to reduce the health and economic burden of CVD [7].

Compared to clinic blood pressure (BP), emerging evidence has consistently demonstrated that 24-hour ambulatory blood pressure monitoring (ABPM) provides superiority in predicting targeted organ damage and cardiovascular events in hypertensive populations [8-11]. The underly-

\author{
Corresponding author: \\ Siping Dai \\ Department of Emergency \\ The Third People's \\ Hospital of Huizhou \\ 1 Qiaodongxuebei St \\ 516000 Huizhou, China \\ Phone: +86752 2255120 \\ E-mail: daisiping123@gmail. \\ com
}


ing mechanisms are multifactorial. For example, ABPM offers more complete and comprehensive $\mathrm{BP}$ information in a longer duration. In addition, $A B P M$ can distinguish hypertensive patterns in terms of the dipping and non-dipping BP pattern within a 24-hour circadian cycle [12, 13].

Prior observational studies indicated that compared to the dipping BP pattern (mean nighttime/ daytime $\mathrm{BP}$ ratio $\leq 0.9$ ), the non-dipping BP pattern (mean nighttime/daytime BP ratio > 0.9) [14] was more closely associated with CVD risks $[15,16]$. Therefore, one might anticipate that in dyslipidemia patients with hypertension, those with the non-dipping BP pattern might also have higher CVD risk compared to their counterparts with the dipping BP pattern. In the present study, we used a cross-sectional design to compare the prevalence of CVD between dyslipidemia patients with dipping and non-dipping BP patterns. From a clinical perspective, such information will help in determining whether correcting the BP pattern would have potential for reducing CVD risk in dyslipidemic patients with hypertension in the future.

\section{Material and methods}

\section{Study populations}

The present study was approved by the Clinical Ethic Committee of the Third People's Hospital of Huizhou and informed consent was obtained before enrollment. Inclusion criteria were as follows: participants had a documented diagnosis of dyslipidemia (based on either fasting serum low-density lipoprotein cholesterol (LDL-C) level $\geq 3.4 \mathrm{mmol} / \mathrm{l}$ or treatment with statins) and hypertension (based on either clinic systolic and/or diastolic BP (SBP/DBP) $\geq 140 / 90 \mathrm{~mm} \mathrm{Hg}$ or treatment with anti-hypertensive medications). Exclusion criteria were as follows: subjects did not want to participate in the current study, or had secondary hypertension, familial dyslipidemia, had acute myocardial infarction, ischemic stroke, or congestive heart failure in the last 12 months.

\section{Collection of clinical and laboratory characteristics}

Patients' characteristics including age, gender, smoking status, body mass index (BMI, weight in kilograms divided by height in squared meters), and medical histories including diabetes mellitus (DM), coronary heart disease (CHD), ischemic stroke (IS) and chronic kidney disease (CKD) were recorded. Current medication usage was also recorded. Laboratory indexes including fasting serum levels of lipid profiles, glucose and creatinine were extracted from electronic medical documents. All these procedures were performed by two independent investigators.

\section{Parameters of clinic BP and ABPM collection}

Clinic BP parameters including SBP and DBP were measured after patients sat quietly for 10 min and three BP readings were obtained at 1 -minute intervals, and the last two $B P$ readings were averaged as clinic BP. ABPM parameters including 24-hour SBP and DBP, and daytime (from 8:00 to 22:00) and nighttime (from 22:00 to 8:00) SBP and DBP were collected. In brief, the device used for ABPM measurement was model no. 90207 (Spacelabs Medical Inc, Redmond, Washington, USA). Ambulatory BP was recorded in the non-dominant arm at 20 min intervals during the daytime and at 30 min intervals during the nighttime. Accordingly, patients with a night-day ratio of SBP and/or DBP $\leq 0.90$ were defined as having a dipping pattern, and those with a ratio $>0.90$ were defined as having a non-dipping pattern [16].

\section{Statistical analysis}

Continuous variables were described using mean \pm SD and categorical variables were described by the number and frequency of cases. The statistical significance of difference was analyzed using Student's $t$-test for continuous variables and the $\chi^{2}$ or Fisher exact test for categorical variables. Logistic regression analysis was used to evaluate the associations between dipping and non-dipping hypertension and the prevalence of CVD including CHD and IS. Statistical analysis was computed using SPSS 17.0 (SPSS Inc, Chicago, $\mathrm{IL})$. All of the statistical tests were two-sided and results were considered statistically significant when the $p$-value $<0.05$.

\section{Results}

\section{Comparisons between dipping}

and non-dipping hypertensive patients

Clinical characteristics, medications usage and laboratory indices of dipping and non-dipping hypertensive patients are listed in Table I. The mean durations of hypertension in both groups were $5.3 \pm 2.1$ years and $5.6 \pm 2.4$ years, respectively. Compared to the dipping group, patients in the non-dipping group were older, and more likely to be male, smokers, and had a higher serum creatinine level and higher prevalence of CKD $(p<$ 0.05 for all comparisons). Additionally, patients in the non-dipping group also more likely had CHD (39.1\% vs. $31.4 \%, p<0.05)$ and CVD (49.3\% vs. $40.9 \%, p<0.05)$. No significant between-group differences in current medications usage including the mean numbers and classes of anti-hypertensive medications and lipid profiles were observed. 
Table I. Comparisons between dipping and non-dipping hypertensive patients

\begin{tabular}{|c|c|c|c|}
\hline Characteristics & Dipping $(n=105)$ & Non-dipping $(n=138)$ & $P$-value \\
\hline \multicolumn{4}{|l|}{ Demographics: } \\
\hline Age [years] & $54.5 \pm 10.8$ & $59.3 \pm 13.6$ & 0.006 \\
\hline Male, $n(\%)$ & $55(52.4)$ & $83(60.1)$ & 0.018 \\
\hline Smoking, $n$ (\%) & 49 (46.7) & $68(49.3)$ & 0.046 \\
\hline $\mathrm{BMI}\left[\mathrm{kg} / \mathrm{m}^{2}\right]$ & $23.7 \pm 2.6$ & $24.1 \pm 3.1$ & 0.270 \\
\hline CHD, $n(\%)$ & $33(31.4)$ & $54(39.1)$ & 0.002 \\
\hline IS, $n(\%)$ & $10(9.5)$ & $14(10.1)$ & 0.722 \\
\hline CVD, $n(\%)$ & $43(40.9)$ & $68(49.3)$ & 0.004 \\
\hline $\mathrm{DM}, n(\%)$ & $27(25.7)$ & $36(26.1)$ & 0.383 \\
\hline CKD, $n(\%)$ & $10(9.5)$ & $17(12.3)$ & 0.039 \\
\hline Hypertension duration [years] & $5.3 \pm 2.1$ & $5.6 \pm 2.4$ & 0.106 \\
\hline \multicolumn{4}{|l|}{ Medications: } \\
\hline Anti-platelet, $n$ (\%) & $64(61.0)$ & $89(64.5)$ & 0.063 \\
\hline Statins, $n$ (\%) & $57(54.3)$ & $78(56.5)$ & 0.109 \\
\hline Anti-hypertension, $n(\%)$ : & 96 (91.4) & $127(92.0)$ & 0.820 \\
\hline $\mathrm{ACEi} / \mathrm{ARB}$ & $68(64.8)$ & $90(65.2)$ & 0.446 \\
\hline $\mathrm{CCB}$ & $22(21)$ & $28(20.3)$ & 0.560 \\
\hline Diuretic & $37(35.2)$ & $52(37.7)$ & 0.094 \\
\hline$\beta$-blocker & $16(15.2)$ & $22(15.9)$ & 0.306 \\
\hline$\alpha$-blocker & $5(4.8)$ & $9(6.5)$ & 0.113 \\
\hline Numbers of agents & $2.4 \pm 0.7$ & $2.6 \pm 0.6$ & 0.645 \\
\hline Anti-diabetes, $n(\%)$ & $27(25.7)$ & $34(24.6)$ & 0.558 \\
\hline \multicolumn{4}{|l|}{ Laboratory indexes: } \\
\hline $\mathrm{FPG}[\mathrm{mmol} / \mathrm{l}]$ & $6.0 \pm 0.9$ & $5.8 \pm 0.7$ & 0.407 \\
\hline $\mathrm{TC}[\mathrm{mmol} / \mathrm{l}]$ & $5.3 \pm 0.5$ & $5.4 \pm 0.8$ & 0.624 \\
\hline LDL-C [mmol/l] & $3.1 \pm 0.5$ & $3.2 \pm 0.6$ & 0.375 \\
\hline $\mathrm{HDL}-\mathrm{C}[\mathrm{mmol} / \mathrm{l}]$ & $1.1 \pm 0.3$ & $1.1 \pm 0.4$ & 0.860 \\
\hline $\mathrm{TG}[\mathrm{mmol} / \mathrm{l}]^{\#}$ & $1.6(1.2-3.7)$ & $1.8(1.1-3.9)$ & 0.443 \\
\hline Creatinine $[\mu \mathrm{mol} / \mathrm{l}]$ & $90.8 \pm 11.6$ & $102.4 \pm 12.2$ & 0.017 \\
\hline
\end{tabular}

FPG - fasting plasma glucose, TC - total cholesterol, LDL-C - low-density lipoprotein cholesterol, HDL-C - high-density lipoprotein cholesterol, TG - triglyceride, CVD - CHD plus IS, "TG - median and interquartile, ACEi/ARB - angiotensin converting enzyme inhibitor/ angiotensin receptor blocker, CCB - calcium channel blocker.

Clinic BP and ABPM comparisons in dipping and non-dipping groups

Parameters of clinic BP and ABPM were compared according to the dipping and non-dipping BP patterns. As summarized in Table II, no significant between-group differences in the clinic SBP and DBP were observed. However, compared to the dipping group, 24-hour SBP, nighttime SBP and DBP, night-day ratio of SBP and DBP were all sig- nificantly higher in the non-dipping group versus the dipping group ( $p<0.05$ for all comparisons).

\section{Associations of BP patterns and prevalence of CVD}

Associations of BP patterns and prevalence of CVD were evaluated by logistic regression analysis. As shown in Table III, the associations of nightday ratios of SBP and DBP with prevalent CVD 
Table II. Clinic BP and ABPM comparisons in dipping and non-dipping groups

\begin{tabular}{|c|c|c|c|}
\hline Characteristics & Dipping $(n=105)$ & Non-dipping $(n=138)$ & $P$-value \\
\hline \multicolumn{4}{|l|}{ Clinic: } \\
\hline $\mathrm{SBP}[\mathrm{mm} \mathrm{Hg}]$ & $134 \pm 17$ & $131 \pm 20$ & 0.755 \\
\hline $\mathrm{DBP}[\mathrm{mm} \mathrm{Hg}]$ & $80 \pm 12$ & $82 \pm 15$ & 0.406 \\
\hline \multicolumn{4}{|l|}{ ABPM: } \\
\hline \multicolumn{4}{|l|}{ 24-hour: } \\
\hline $\mathrm{SBP}[\mathrm{mm} \mathrm{Hg}]$ & $120 \pm 13$ & $126 \pm 16$ & 0.023 \\
\hline $\mathrm{DBP}[\mathrm{mm} \mathrm{Hg}]$ & $74 \pm 12$ & $74 \pm 10$ & 0.630 \\
\hline \multicolumn{4}{|l|}{ Daytime: } \\
\hline $\mathrm{SBP}[\mathrm{mm} \mathrm{Hg}]$ & $126 \pm 15$ & $125 \pm 13$ & 0.843 \\
\hline $\mathrm{DBP}[\mathrm{mm} \mathrm{Hg}]$ & $76 \pm 9$ & $75 \pm 12$ & 0.308 \\
\hline \multicolumn{4}{|l|}{ Nighttime: } \\
\hline $\mathrm{SBP}[\mathrm{mm} \mathrm{Hg}]$ & $114 \pm 11$ & $120 \pm 10$ & $<0.001$ \\
\hline $\mathrm{DBP}[\mathrm{mm} \mathrm{Hg}]$ & $69 \pm 8$ & $73 \pm 10$ & 0.003 \\
\hline \multicolumn{4}{|l|}{ Night-day ratio: } \\
\hline SBP & $0.83 \pm 0.04$ & $0.97 \pm 0.05$ & $<0.001$ \\
\hline DBP & $0.81 \pm 0.07$ & $0.95 \pm 0.04$ & $<0.001$ \\
\hline
\end{tabular}

SBP - systolic blood pressure, DBP - diastolic blood pressure, ABPM - ambulatory blood pressure monitoring.

Table III. Association of BP pattern and prevalence of CVD

\begin{tabular}{|c|c|c|c|c|}
\hline \multicolumn{2}{|l|}{ Model } & Odds ratio & $95 \%$ confidence interval & $P$-value \\
\hline \multicolumn{5}{|c|}{ Dipping pattern: } \\
\hline \multirow[t]{2}{*}{ Model 1} & Night-day ratio of SBP & 1.34 & $1.18-1.96$ & $<0.001$ \\
\hline & Night-day ratio of DBP & 1.16 & $0.94-1.63$ & 0.089 \\
\hline \multirow[t]{2}{*}{ Model 2} & Night-day ratio of SBP & 1.18 & $1.09-1.46$ & 0.007 \\
\hline & Night-day ratio of DBP & & & NS \\
\hline \multirow[t]{2}{*}{ Model 3} & Night-day ratio of SBP & 1.09 & $1.02-1.34$ & 0.011 \\
\hline & Night-day ratio of DBP & & & NS \\
\hline \multicolumn{5}{|c|}{ Non-dipping pattern: } \\
\hline \multirow[t]{2}{*}{ Model 1} & Night-day ratio of SBP & 2.01 & $1.47-2.69$ & $<0.001$ \\
\hline & Night-day ratio of DBP & 1.45 & $1.17-1.85$ & 0.006 \\
\hline \multirow[t]{2}{*}{ Model 2} & Night-day ratio of SBP & 1.89 & $1.43-2.11$ & $<0.001$ \\
\hline & Night-day ratio of DBP & 1.36 & $1.17-1.90$ & 0.014 \\
\hline \multirow[t]{2}{*}{ Model 3} & Night-day ratio of SBP & 1.72 & $1.33-2.06$ & $<0.001$ \\
\hline & Night-day ratio of DBP & 1.23 & $1.05-1.66$ & 0.033 \\
\hline
\end{tabular}

Model 1 adjusted for age and gender; Model 2 additionally adjusted for smoking, BMI, DM, CKD and LDL-C; Model 3 adjusted for clinic SBP and DBP; NS - non-significant.

were assessed in dipping and non-dipping groups, respectively. In the dipping group, only night-day ratio of SBP was significantly associated with prevalent CVD after adjusting for potential risk factors, with an odds ratio (OR) of 1.09 (95\% confidence interval (CI) of 1.02-1.34). Nevertheless, in the non-dipping group, both night-day ratio of SBP and DBP were significantly associated with 
prevalent CVD after being extensively adjusted for potential risk factors including clinic SBP and DBP, with an OR of $1.72(95 \% \mathrm{Cl}: 1.33-2.06)$ and 1.23 (95\% Cl: 1.05-1.66), respectively.

\section{Discussion}

Our present study shows that in dyslipidemia patients with hypertension, the night-day ratio of SBP and DBP measured by 24-hour ABPM could be used to complement clinic SBP and DBP in evaluating CVD risk. In addition, the non-dipping BP pattern appears to be more closely related to prevalent CVD compared to the dipping BP pattern in dyslipidemia patients. Future randomized controlled trials are warranted to investigate whether correcting the BP pattern will reduce cardiovascular events in dyslipidemia patients with hypertension. Specifically, we can enroll dyslipidemia patients with the non-dipping BP pattern and randomly assign them to an active group and a placebo group to evaluate whether reversing the non-dipping BP pattern by using nighttime anti-hypertensive medications would reduce cardiovascular events compared to the placebo group.

Owing to the high proportion of population attributable risk accounted for both dyslipidemia and hypertension [2], it is clinically relevant to investigate whether different BP patterns would have differing effects on cardiovascular systems in patients with dyslipidemia. Notably, the non-dipping BP pattern is better than the dipping BP pattern in relation to cardiovascular events in general hypertensive patients [17, 18]. Consistent with previous findings, our present study also showed that in patients with dyslipidemia, the non-dipping BP pattern was better than the dipping BP pattern in relation to prevalent CVD after extensively adjusting for traditional risk factors including age, smoking, lipid profiles, DM, CKD, as well as clinic SBP and DBP. The following possibilities might be ascribed to these findings. First, blunted $\mathrm{BP}$ reduction during nighttime is commonly associated with enhanced sympathetic activity, which in turn increases heart rate and cardiac output [19-21]. Of note, these hemodynamic alterations are detrimental to cardiovascular systems, thereby increasing CVD risk. Second, obstructive sleep apnea (OSA) is closely associated with non-dipping hypertension. It is known that OSA is significantly associated with systemic inflammation, oxidative stress and endothelial dysfunction [19]. Therefore, it is conceivable that the effects of the non-dipping hypertensive pattern exerted on cardiovascular systems might be partially associated with unrecognized OSA. Third, the predictive value of daytime BP may be attenuated by its higher variability as compared to nighttime BP [20-22], which renders nighttime BP measured by 24-hour ABPM essential for CVD risk discrimination.

We observed significant differences in clinical characteristics between dipping and non-dipping BP pattern groups. For example, patients with the non-dipping pattern were older and more likely to be male and smokers and had higher prevalence of CKD, suggesting that these patients per se might be at a higher CVD risk than their dipping counterparts. We also observed that clinic SBP and DBP were comparable between the dipping and non-dipping groups. Nevertheless, there were significant differences in 24-hour BP parameters between these two groups. As presented in Table II, both nighttime SBP and DBP were significantly higher in the non-dipping group, while no significant between-group differences were observed in daytime SBP and DBP, further supporting the notion that nighttime BP might be more closely associated with CVD than daytime BP [16], and 24-hour ABPM is essential for dyslipidemia patients with hypertension in terms of CVD risk assessment [23].

There are several limitations of our present study. First, the cross-sectional design could not allow us to infer a causal relationship between non-dipping BP pattern and prevalent CVD in patients with dyslipidemia. However, emerging evidence has shown that blunted nocturnal BP fall was associated with greater CVD risk. Second, the relatively small number of IS cases might not allow us to observe any significant difference between the dipping and non-dipping groups. However, owing to the similar pathophysiological mechanisms underlying CHD and IS, it was possible that the non-dipping pattern was more closely related to IS than the dipping pattern. Third, as we have mentioned, OSA is highly prevent in non-dipping hypertensive patients. However, we did not evaluate OSA in the current study. Therefore, we could not adjust OSA for the association of non-dipping BP pattern and prevalent CVD. In our ongoing study, we will expand our studied sample size and evaluate OSA prospectively, so as to evaluate the effects of OSA on BP pattern change at night as well as to evaluate the association between non-dipping BP pattern and CVD risk in dyslipidemic patients with adjustment for OSA. Last but not least, our participants had comorbidities such as CHD, IS, diabetes mellitus and CKD, which might somewhat influence BP pattern and its association with prevalent CVD. Prospective cohort studies are needed to evaluate whether BP pattern can be used to predict the future incidence of CVD in patients with dyslipidemia but without prevalent CVD at baseline.

In conclusion, our present study reveals that in patients with dyslipidemia, the non-dipping 
$\mathrm{BP}$ pattern is better than the dipping pattern in relation to prevalent CVD, and 24-hour ABPM measurement is better than clinic $\mathrm{BP}$ in relation to prevalent CVD. Future research is necessary to investigate whether reversing the non-dipping pattern will reduce the CVD risk in patients with dyslipidemia.

\section{Acknowledgments}

We are indebted to Dr. Ying Wang for her help in conducting statistical analysis for our paper.

Current study was supported from The Technology Grant of Huizhou (170512101740855).

\section{Conflict of interest}

The authors declare no conflict of interest.

\section{References}

1. Fihn SD, Gardin JM, Abrams J, et al, American College of Cardiology Foundation/American Heart Association TaskF.2012ACCF/AHA/ACP/AATS/PCNA/SCAI/STSguideline for the diagnosis and management of patients with stable ischemic heart disease: a report of the American College of Cardiology Foundation/American Heart Association task force on practice guidelines, and the American College of Physicians, American Association for Thoracic Surgery, Preventive Cardiovascular Nurses Association, Society for Cardiovascular Angiography and Interventions, and Society of Thoracic Surgeons. Circulation 2012; 126: e354-471.

2. Yusuf S, Hawken S, Ounpuu S, et al. Effect of potentially modifiable risk factors associated with myocardial infarction in 52 countries (the Interheart Study): case-control study. Lancet 2004; 364: 937-52.

3. Kearney PM, Whelton M, Reynolds K, Muntner P, Whelton PK, He J. Global burden of hypertension: analysis of worldwide data. Lancet 2005; 365: 217-23.

4. Horwich TB, Hamilton MA, Maclellan WR, Fonarow GC. Low serum total cholesterol is associated with marked increase in mortality in advanced heart failure. J Cardiac Failure 2002; 8: 216-24.

5. Awad K, Serban MC, Penson P, et al. Effects of morning vs evening statin administration on lipid profile: a systematic review and meta-analysis. J Clin Lipidol 2017; 11: 972-85 e979.

6. Zanchetti A, Liu L, Mancia G, et al. Blood pressure and LDL-cholesterol targets for prevention of recurrent strokes and cognitive decline in the hypertensive patient: design of the European Society of Hypertension-Chinese Hypertension League Stroke in hypertension optimal treatment randomized trial. J Hypertens 2014; 32: 1888-97.

7. Zanchetti A, Liu L, Mancia G, et al. Blood pressure and low-density lipoprotein-cholesterol lowering for prevention of strokes and cognitive decline: a review of available trial evidence. J Hypertens 2014; 32: 1741-50.

8. Clement DL, De Buyzere ML, De Bacquer DA, et al.; Office versus Ambulatory Pressure Study I. Prognostic value of ambulatory blood-pressure recordings in patients with treated hypertension. N Engl J Med 2003; 348: 2407-15.

9. Boggia J, Li Y, Thijs L, et al.; International Database on Ambulatory blood pressure monitoring in relation to
Cardiovascular Outcomes I. Prognostic accuracy of day versus night ambulatory blood pressure: a cohort study. Lancet 2007; 370: 1219-29.

10. Yano Y, Kario K. Nocturnal blood pressure and cardiovascular disease: a review of recent advances. Hypertens Res 2012; 35: 695-701.

11. Ghomari-Boukhatem H, Bouchouicha A, Mekki K, Chenni K, Belhadj M, Bouchenak M. Blood pressure, dyslipidemia and inflammatory factors are related to body mass index in scholar adolescents. Arch Med Sci 2017; 13: 46-52.

12. de la Sierra A, Redon J, Banegas JR, et al.; Spanish Society of Hypertension Ambulatory Blood Pressure Monitoring Registry I. Prevalence and factors associated with circadian blood pressure patterns in hypertensive patients. Hypertension 2009; 53: 466-72.

13. Dolan E, Stanton A, Thijs L, et al. Superiority of ambulatory over clinic blood pressure measurement in predicting mortality: the Dublin Outcome Study. Hypertension 2005; 46: 156-61.

14. Parati G, Stergiou G, O'Brien E, et al. European Society of Hypertension practice guidelines for ambulatory blood pressure monitoring. J Hypertens 2014; 32: 1359-66.

15. Fagard RH, Celis H, Thijs L, et al. Daytime and nighttime blood pressure as predictors of death and cause-specific cardiovascular events in hypertension. Hypertension 2008; 51: 55-61.

16. Wei FF, Li Y, Zhang L, et al. Association of target organ damage with 24-hour systolic and diastolic blood pressure levels and hypertension subtypes in untreated Chinese. Hypertension 2014; 63: 222-8.

17. Staessen JA, Thijs L, Fagard R, et al.; Investigator SHET. Predicting cardiovascular risk using conventional vs ambulatory blood pressure in older patients with systolic hypertension. JAMA 1999; 282: 539-46.

18. Parati G, Stergiou G, O'Brien E, et al.; European Society of Hypertension Working Group on Blood Pressure M, Cardiovascular V. European Society of Hypertension practice guidelines for ambulatory blood pressure monitoring. J Hypertens 2014; 32: 1359-66.

19. Cai A, Wang L, Zhou Y. Hypertension and obstructive sleep apnea. Hypertens Res 2016; 39: 391-5.

20. Ohkubo T, Hozawa A, Yamaguchi J, et al. Prognostic significance of the nocturnal decline in blood pressure in individuals with and without high 24-h blood pressure: the Ohasama Study. J Hypertens 2002; 20: 2183-9.

21. Stulc T, Lanska V, Snejdrlova M, Vrablik M, Prusikova M, Ceska R. A comprehensive guidelines-based approach reduces cardiovascular risk in everyday practice: the VARO Study. Arch Med Sci 2017; 13: 705-10.

22. Harris MA, Ferguson TS, Boyne MS, Figueroa JP. High prevalence of dyslipidemia among primary care patients with hypertension and diabetes in Jamaica. Arch Med Sci Atheroscler Dis 2017; 2: e61-7.

23. Wang Y, Ma H, Yang J, Chen O, Lu L, Zhang R. Lipoprotein(a) is associated with left ventricular systolic dysfunction in a Chinese population of patients with hypertension and without coronary artery disease. Arch Med Sci 2017; 13: 1078-85. 